\title{
Successful Local Anesthesia for Restorative Dentistry and Endodontics
}

Authors: Al Reader / John Nusstein / Melissa Drum

Publisher: Quintessence Publishing, USA

Language: English

ISBN: 978-0-86715-743-7

Edition: $2 / \mathrm{e}$

Publish Year: 2017

Pages: 240 , illustrated

Price: $88.00 €$

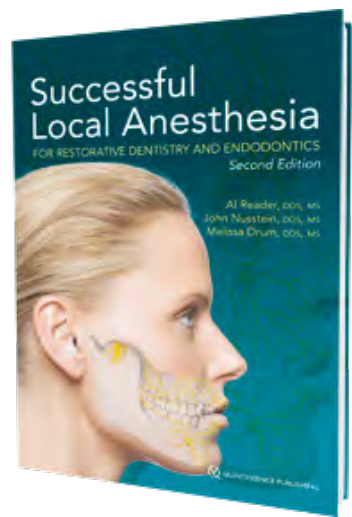

It is known that besides the fear of pain, which is the main factor that makes patients delay the visit to the dentist one should add also the fact that $90 \%$ of the dentists encounter some difficulties while performing anesthesia during restorative dental procedures. For the success of local anesthesia in dental and endodontic restorations, the endodontic team of College of Dentistry's Division at The Ohio State University, Columbus, Ohio, has offered practitioners this book to ensure patient anesthesia by using the latest medicines and techniques. The book is divided in seven chapters. The first chapter discusses the clinical factors of dental anesthesia such as: confirming pulpal anesthesia in nonpainful vital teeth, clinical local anesthesia, anxiety and pain, use of vasoconstrictors, characterize of injection pain, use of topical anesthetic alternative mode of reducing injection pain and alternative modes. Mandibular anesthesia is the subject of the second chapter, which tackles the inferior alveolar nerve block (IANB), anesthetic solution for IANB, mandibular injection locations, attempts to increase success of IANB in asymptomatic patients, mechanism of failure with the IANB, why asymptomatic patients do not achieve pulpal anesthesia with the IANB and methods to increase the success of mandibular anesthesia. The third chapter presents maxillary anesthesia by infiltration injection, it lists alternative anesthetic solutions, increasing the duration of pulpal anesthesia for infiltrations and discribes alternate injections techniques. Supplemental anesthesia is the subject of Chapter 4, which describes supplemental infiltration, intraligamentar and intraosseous injections, indication and success rates. The fifth chapter talks about the clinical tips for the management of routine restorative procedures and provides information on anesthetic success for molars, premolars and anterior teeth, mandibular and maxillary. Endodontic anesthesia is presented in Chapter 6, detailing the clinical factors and methods related to confirming pulpal anesthesia, factors related to pain, the success of local anesthesia in irreversible pulpitis, postoperative pain reduction in irreversible pulpitis, the use of supplemental intraligamentary, intraosseous, intraseptal and intrapulpar injection. The last chapter describes some clinical tips for the management of specific endododntic situations describing how to successfully anesthetize and defines some considerations regarding endodontic anesthesia. The book is accompanied by an index and each chapter benefits from abundant references. The book guides the practitioner on how to make a successful anesthesia using the latest techniques and drugs; the book is a guide that should not be missing from the library of every practitioner who is aiming at dental and endodontic high-quality restorations.

DOI: 10.25241/stomaeduj.2018.5(1).bookreview.2

Marian-Vladimir Constantinescu

$\mathrm{DDS}, \mathrm{PhD}$

Holistic Dental \& Medical Institute of Bucharest-ROPOSTURO, Bucharest Romania e-mail: dr.vladimir.constantinescu@gmail.com

The Books Review is drafted in the reviewer's sole wording and illustrates his opinions. 